\title{
Properties of $\mathrm{BaTiO}_{3} / \mathrm{BaZrO}_{3}$ ferroelectric superlattices with competing instabilities
}

\author{
Alexander I. Lebedev* \\ Physics Department, Moscow State University, \\ Leninskie gory, 119991 Moscow, Russia
}

(Dated: November 5, 2018)

\begin{abstract}
Properties of $\left(\mathrm{BaTiO}_{3}\right)_{1} /\left(\mathrm{BaZrO}_{3}\right)_{n}$ ferroelectric superlattices (SLs) with $n=1-7$ grown in the [001] direction are calculated from first principles within the density functional theory. It is revealed that the quasi-two-dimensional ferroelectricity occurs in these SLs in the barium titanate layers with a thickness of one unit cell; the polarization is oriented in the layer plane and weakly interacts with the polarization in neighboring layers. The ferroelectric ordering energy and the height of the barrier separating different orientational states of polarization in these SLs are sufficiently large to provide the formation of an array of independent polarized planes at $300 \mathrm{~K}$. The effect of the structural instability on the properties of SLs is considered. It is shown that the ground state is a result of simultaneous condensation of the $\Gamma_{15}$ polar phonon and phonons at the $M$ point (for SLs with even period) or at the $A$ point (for SLs with odd period); it is a polar structure with out-of-phase rotations of the octahedra in neighboring layers, in which highly polarized layers are spatially separated from the layers with strong rotations. The competition between the ferroelectric and structural instabilities in biaxially compressed SLs manifests itself in that the switching on of the octahedra rotations leads to an abrupt change of the polarization direction and can cause an improper ferroelectric phase transition to occur. It was shown that the experimentally observed $z$ component of polarization in the SLs can appear only as a result of the mechanical stress relaxation.
\end{abstract}

PACS numbers: 63.22.-m, 68.65.Cd, 77.84.Cg, 81.05.Zx

\section{INTRODUCTION}

Ferroelectric superlattices (SLs) represent a new class of artificial materials whose properties, such as the Curie temperature and spontaneous polarization, often exceed those of bulk ferroelectrics $: 1,2$ An analysis of properties of ten ferroelectric SLs with the perovskite structure grown in the [001] direction have shown that they are ferroelectrics at low temperatures, no matter whether they are composed of two ferroelectrics, a ferroelectric and a paraelectric, or even two paraelectrics $\frac{3}{3}$ One of the possible applications of SLs can be non-volatile ferroelectric random access memory (FRAM) devices in electronics.

The recent studies of the ferroelectric instability in free-standing $\left(\mathrm{KNbO}_{3}\right)_{1}\left(\mathrm{KTaO}_{3}\right)_{n}$ superlattices grown in the [001] direction showed that, with increasing $n$, the tendency to the ferroelectric ordering is retained in $\mathrm{KNbO}_{3}$ layers with a thickness of one unit cell while the interaction energy between neighboring $\mathrm{KNbO}_{3}$ layers exponentially decreases. $\frac{4.5}{.5}$ It was shown that at $n \geq 2$ the ground state of the SLs is an array of ferroelectrically polarized planes whose polarization is concentrated in the $\mathrm{KNbO}_{3}$ layer, is parallel to the [110] direction, and weakly interacts with polarization in neighboring layers. The use of such arrays of quasi-two-dimensional ferroelectric planes as a medium for the three-dimensional data recording can make it possible to achieve a volume recording density of $\sim 10^{18} \mathrm{bit} / \mathrm{cm}^{3}$.

Unfortunately, the implementation of the abovedescribed ground state in the $\left(\mathrm{KNbO}_{3}\right)_{1}\left(\mathrm{KTaO}_{3}\right)_{n}$ SLs requires very low temperatures $(\sim 4 \mathrm{~K})$ because of the low ferroelectric ordering energy and low height of the potential barrier separating different orientational states of polarization. That is why one of the aims of this work was to study the feasibility of similar quasi-two-dimensional structures and the ways to improve their characteristics in $\mathrm{BaTiO}_{3} / \mathrm{BaZrO}_{3}$ ferroelectric superlattices with an active layer of barium titanate.

In $\mathrm{BaTiO}_{3} / \mathrm{BaZrO}_{3}$ superlattices, the tension of the $\mathrm{BaTiO}_{3}$ layers as a result of the epitaxial matching with the $\mathrm{BaZrO}_{3}$ layers having a larger lattice parameter should stabilize the Amm2 ground state necessary to implement the quasi-two-dimensional structure ${ }^{4}$ in the $\mathrm{BaTiO}_{3}$ layers. However, since the simultaneous compression of the $\mathrm{BaZrO}_{3}$ layers can cause the polarization normal to the interface to occur in them,,$\frac{6}{-}$ the search for the ground-state structure in these SLs requires a detailed analysis. Furthermore, the instability of the $\mathrm{BaZrO}_{3}$ structure with respect to the octahedra rotation $^{7-9}$ can result in the competition of the ferroelectric and structural instabilities in the SLs, and this problem also calls for a detailed study.

The properties of $\mathrm{BaTiO}_{3} / \mathrm{BaZrO}_{3}$ SLs have been studied previously both experimentally $\underline{\underline{10}} \underline{-15}$ and theoretically. $\frac{3}{\underline{3}}$ The initial interest to these SLs was caused by giant dielectric constants observed in them. 10 Roomtemperature observations of ferroelectric hysteresis loops in the SLs $11,13,15$ suggested that the ferroelectric transition temperature is them exceeds $300 \mathrm{~K}$. The spontaneous polarization nonmonotonically varied with the period of SLs and depended on the electrode configuration (in short-period SLs with electrodes on both sides of the film, hysteresis loops were absent $\frac{13}{3}$ ). These features have already been discussed in Ref. 3 and were explained by the rotation of the polarization to the layer plane. The recent study of Raman spectra and dielectric properties of $\mathrm{BaTiO}_{3} / \mathrm{BaZrO}_{3} \mathrm{SLs}$ revealed the appearance of the $z$-component of polarization in barium titanate 
layers in the SLs grown on $\mathrm{MgO}$ substrates $\frac{15}{15}$ Interpretation of these results requires the study of the influence of substrate-induced strain on the ground-state structure of the SLs.

\section{CALCULATION TECHNIQUE}

The $\left(\mathrm{BaTiO}_{3}\right)_{1}\left(\mathrm{BaZrO}_{3}\right)_{n}$ superlattices considered in this work are periodic structures grown in the [001] direction and consisting of the $\mathrm{BaTiO}_{3}$ layer with a thickness of one unit cell and the $\mathrm{BaZrO}_{3}$ layer with a thickness of $n$ unit cells $(1 \leq n \leq 7)$. These SLs were modeled on supercells of $1 \times 1 \times \mathrm{L}$ unit cells, where $L=n+1$ is the SL period; in modeling the structures generated by instabilities at the $M$ and $A$ points at the boundary of the Brillouin zone, the primitive cell volume was doubled. In this work, particular attention was paid to free-standing SLs. In addition, a number of calculations were performed for biaxially compressed $\left(\mathrm{BaTiO}_{3}\right)_{1}\left(\mathrm{BaZrO}_{3}\right)_{1}$ and $\left(\mathrm{BaTiO}_{3}\right)_{2}\left(\mathrm{BaZrO}_{3}\right)_{2}$ SLs and for free-standing $\left(\mathrm{BaTiO}_{3}\right)_{n}\left(\mathrm{BaZrO}_{3}\right)_{n}$ SLs with $n=2$, 3 , and 4 .

The calculations were performed using the density functional theory with pseudopotentials and wave function expansion in plane waves as implemented in the ABINIT code ${ }^{16}$ As in the earlier study of these SLs,,$\frac{3}{}$ the exchange-correlation interaction was described in the local density approximation (LDA). As pseudopotentials, the optimized separable nonlocal pseudopotentials constructed using the OPIUM program 17 were used; the local potential correction was added to them to improve the transferability. The parameters used for constructing the pseudopotentials and other calculation details are given in Refs. 3 and 18 . The cut-off energy for the plane waves was $30 \mathrm{Ha}(816 \mathrm{eV})$; the integration over the Brillouin zone was performed using the $8 \times 8 \times 4$ Monkhorst-Pack mesh for SLs with $n=1$ and 2 and the $8 \times 8 \times 2$ one for SLs with $n=3-7$. The relaxation of the lattice parameters and atomic positions was performed until the Hellmann-Feynman forces became less than $5 \cdot 10^{-6} \mathrm{Ha} / \mathrm{Bohr}(0.25 \mathrm{meV} / \AA)$. In the case of biaxially compressed SLs, the in-plane lattice parameter was fixed equal to $0.97 a_{0}, 0.98 a_{0}$, and $0.99 a_{0}$, where $a_{0}=7.7182 \mathrm{Bohr}(4.0843 \AA)$ is the in-plane lattice parameter for the $P 4 / \mathrm{mmm}$ phase of the free-standing $\left(\mathrm{BaTiO}_{3}\right)_{1}\left(\mathrm{BaZrO}_{3}\right)_{1} \mathrm{SL}$. The phonon spectra were calculated using the equations obtained from the density functional perturbation theory. The total spontaneous polarization in the SLs was calculated by the Berry phase method; its distribution over layers was calculated using the formula $P_{\alpha}=\sum_{i} w_{i} Z_{i, \alpha \beta}^{*} u_{i, \beta}$ from displacements $u_{i}$ of atoms obtained in the polar phase and tensors of their effective Born charges $Z_{i, \alpha \beta}^{*}$ in the paraelectric phase; here $w_{i}$ are the weight factors equal to unity for atoms in the $\mathrm{Ti}-\mathrm{O}$ or $\mathrm{Zr}-\mathrm{O}$ layer under consideration, $1 / 2$ for atoms in neighboring $\mathrm{Ba}-\mathrm{O}$ layers, and zero for other atoms.

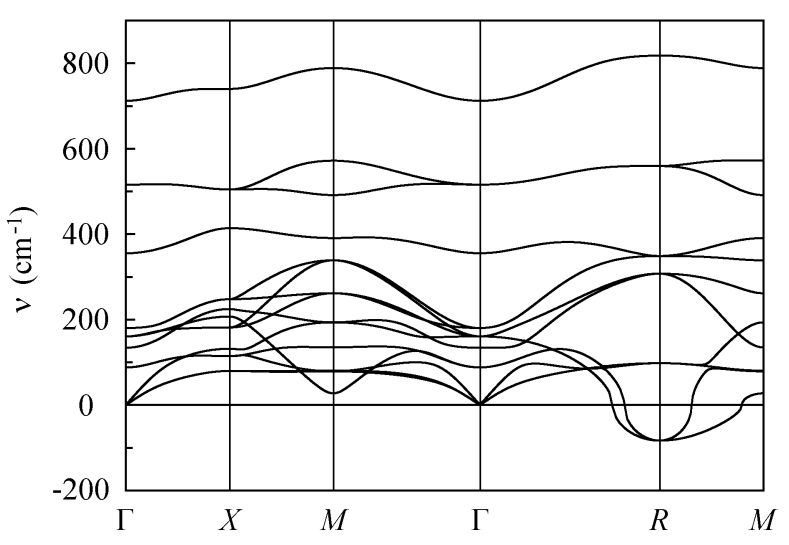

FIG. 1. The phonon spectrum of $\mathrm{BaZrO}_{3}$ in the cubic phase.

The properties of $\mathrm{BaTiO}_{3}$ obtained using the described approach were published previously $\underline{\underline{18}}$ and are in good agreement with experiment.

\section{RESULTS}

\section{A. Structure of $\mathrm{BaZrO}_{3}$}

The structure of $\mathrm{BaZrO}_{3}$ calculated using the described approach is in good agreement with experiment and results of earlier calculations $\underline{\underline{7}-\underline{9}}$ For example, the lattice parameter in cubic $\mathrm{BaZrO}_{3}$ is $7.8724 \mathrm{Bohr}$ $(4.1659 \AA)$ and differs from the experimental value (4.191 $\AA$ at 2 K, Ref. 7) by $0.60 \%$ (the small underestimate of the lattice parameters is characteristic of the used LDA approximation). As in earlier studies, $\underline{\underline{7}-\underline{9}}$ the calculations of the phonon spectrum of cubic $\mathrm{BaZrO}_{3}$ reveal an unstable phonon mode at the $R$ point at the boundary of the Brillouin zone with a frequency of $81 i \mathrm{~cm}^{-1}$ (Fig. 11). The unstable phonon at the $M$ point observed in Ref. 9 is absent in our calculations.

However, the instability of the phonon spectrum of $\mathrm{BaZrO}_{3}$ at the $R$ point has not yet been confirmed experimentally. One of the possible causes of disagreement between experiment and theory can be quantum fluctuations ${ }^{7}$ which can destroy the long-range order in rotations of the oxygen octahedra ${ }^{19}$ According to our calculations, the ground-state structure of $\mathrm{BaZrO}_{3}$ is $I 4 / \mathrm{mcm}$. This result differs from predictions of Ref. 8 , in which the $P \overline{1}$ structure was considered to be equilibrium. In the present study, it was shown that the $P \overline{1}$ structure is nonequilibrium and slowly relaxes to the $I 4 / \mathrm{mcm}$ structure. The calculated static dielectric constant of barium zirconate in the ground state is $\varepsilon_{x x}=\varepsilon_{y y}=58.8$, $\varepsilon_{z z}=53.3$ (the experimental value is 47 , Ref. 7 ). 

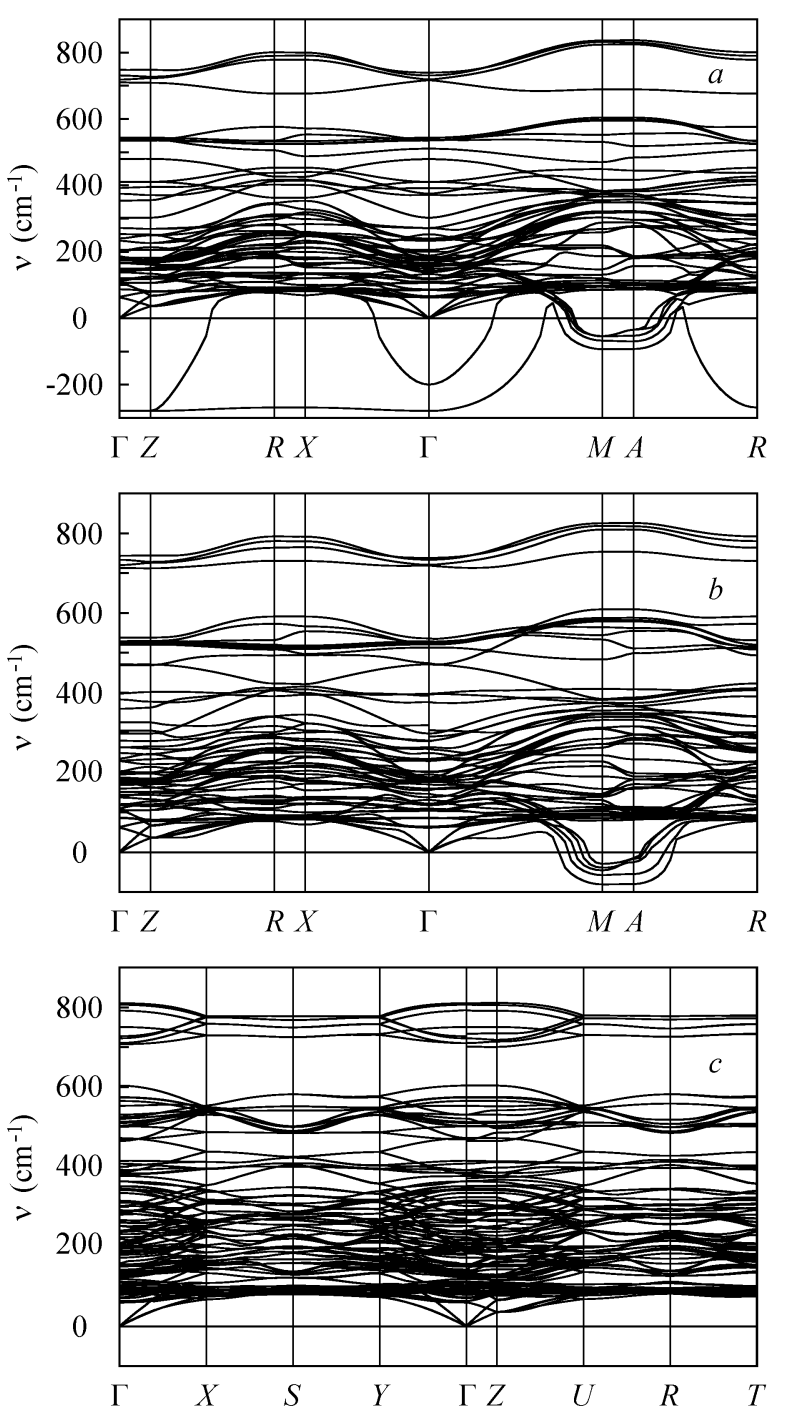

FIG. 2. Phonon spectra of the free-standing $\left(\mathrm{BaTiO}_{3}\right)_{1}\left(\mathrm{BaZrO}_{3}\right)_{3}$ superlattice in (a) P4/mmm, (b) Amm2, and (c) $P m c 2_{1}$ phases.

\section{B. The $\mathrm{BaTiO}_{3} / \mathrm{BaZrO}_{3}$ superlattices}

The phonon spectra of different phases of the freestanding $\left(\mathrm{BaTiO}_{3}\right)_{1}\left(\mathrm{BaZrO}_{3}\right)_{3}$ superlattice are shown in Fig. 2. One can see that two types of instability are observed in the phonon spectrum of the paraelectric P4/mmm phase (Fig. 2 2 a): the ferroelectric instability and the structural instability associated with the oxygen octahedra rotation. The phonon frequencies at highsymmetry points of the Brillouin zone in the paraelectric phase of superlattices with $n=1,2$, and 3 are given in Table [.

The instability region appearing as a band of imaginary phonon frequencies along the path $\Gamma-Z-R-X-\Gamma$ (imaginary frequencies are presented as negative numbers in the figure) is caused by the ferroelectric insta- bility of ...-Ti-O- $-\ldots$ chains, which was established for the first time Ref. 20. An analysis of the eigenvectors of phonons related to this instability region shows that outof-phase transverse $\mathrm{Ti}$ and $\mathrm{O}$ atomic displacements in the $x y$ plane in chains propagating in the [100] and [010] directions dominate in these eigenvectors at all points of the Brillouin zone; at the Brillouin zone center, this displacement pattern corresponds to the doubly degenerate ferroelectric $E_{u}$ mode. For out-of-phase atomic displacements in the chains propagating in the [001] direction and consisting of alternating titanium and zirconium atoms $(\ldots-\mathrm{Ti}-\mathrm{O}-\mathrm{Zr}-\mathrm{O}-\ldots)$, the lowest-energy ferroelectric $A_{2 u}$ mode at the $\Gamma$ point is always stable; its frequency increases from $37 \mathrm{~cm}^{-1}$ for $n=1$ to $85 \mathrm{~cm}^{-1}$ for $n=3$. A similar instability region along the path $\Gamma-Z-R-X-\Gamma$ was observed in $\mathrm{KNbO}_{3} / \mathrm{KTaO}_{3}$ superlattices ${ }^{5}$

Among the possible polar phases resulting from the condensation of the unstable $E_{u}$ mode at the $\Gamma$ point, the $A m m 2$ phase with polarization along the [110] direction has the lowest energy in $\left(\mathrm{BaTiO}_{3}\right)_{1}\left(\mathrm{BaZrO}_{3}\right)_{n}$ SLs with $n=1-7$. However, the phonon spectra of this phase (Fig. 2,b) show that phonons at the $M$ and $A$ points at the boundary of the Brillouin zone remain unstable, which indicates the instability of the $A m m 2$ structure with respect to the oxygen octahedra rotation $\underline{21}$ This instability of the polar $A m m 2$ phases is characteristic of SLs with all periods (see Table \). Thus, the Amm2 phase is not a true ground-state structure for the SLs under consideration.

As follows from Table I the frequencies of two unstable phonons at the $M$ and $A$ points for the $A m m 2$ phase of the superlattices with different periods are very close. Therefore, to search for the true ground state, it is necessary to compare the energies of all structures resulting from the condensation of these two phonons.

An analysis of the eigenvectors of unstable phonons at the $M$ point shows that the most unstable phonons are characterized by out-of-phase rotations of octahedra in neighboring layers. In SLs with even period $(L=2,4)$, the rotation angle is small in the $\mathrm{BaTiO}_{3}$ layers and large in the $\mathrm{BaZrO}_{3}$ layers (Figs. 3 . $a$ and 3 c); in SLs with odd period $(L=3)$, the rotation angle is zero in the $\mathrm{BaTiO}_{3}$ layers and is large in the $\mathrm{BaZrO}_{3}$ layers (Fig. 3. $b$ ). Modes with lower instability (Table I) include modes with other combinations of rotations in layers (in particular, inphase rotations in $\mathrm{BaZrO}_{3}$ layers and strong out-of-phase rotations in every second $\mathrm{BaZrO}_{3}$ layer) and a doubly degenerate mode with a complex combination of distortion and tilting of the oxygen octahedra (for $L=4$ ).

An analysis of the eigenvectors of unstable phonons at the $A$ point shows that in SLs with even period $(L=2$, 4 ), the most unstable phonons are characterized by the out-of-phase rotations in neighboring $\mathrm{BaZrO}_{3}$ layers and the absence of rotations in the $\mathrm{BaTiO}_{3}$ layers (Figs. 3 , $d$ and $3, f)$. In SLs with odd period $(L=3)$, out-of-phase rotation angles are large in the $\mathrm{BaZrO}_{3}$ layers and small in the $\mathrm{BaTiO}_{3}$ layers (Fig. 3. e). In both cases, the physical period of SLs is doubled in comparison with the period 
TABLE I. The lowest frequencies of optical phonons at high-symmetry points of the Brillouin zone in $P 4 / m m m, A m m 2$ phases, and in the ground state $\left(P m c 2_{1}\right)$ of free-standing $\left(\mathrm{BaTiO}_{3}\right)_{1}\left(\mathrm{BaZrO}_{3}\right)_{n}$ superlattices with $n=1,2$, and 3 .

\begin{tabular}{|c|c|c|c|c|c|c|}
\hline \multirow[t]{2}{*}{$n$} & \multicolumn{6}{|c|}{ Phonon frequencies $\left(\mathrm{cm}^{-1}\right)$} \\
\hline & $\Gamma(0,0,0)$ & $Z\left(0,0, \frac{1}{2}\right)$ & $X\left(\frac{1}{2}, 0,0\right)$ & $R\left(\frac{1}{2}, 0, \frac{1}{2}\right)$ & $M\left(\frac{1}{2}, \frac{1}{2}, 0\right)$ & $A\left(\frac{1}{2}, \frac{1}{2}, \frac{1}{2}\right)$ \\
\hline & \multicolumn{6}{|c|}{ The $P 4 / m m m$ phase } \\
\hline 1 & $248 i$ & $243 i$ & $233 i$ & $228 i$ & $90 i$ & $86 i$ \\
\hline 2 & $269 i$ & $268 i$ & $257 i$ & $257 i$ & $88 i, 65 i$ & $90 i, 63 i, 21 i$ \\
\hline \multirow[t]{2}{*}{3} & $279 i$ & $279 i$ & $268 i$ & $268 i$ & $92 i, 66 i, 54 i, 53 i$ & $91 i, 68 i, 51 i, 34 i$ \\
\hline & \multicolumn{6}{|c|}{ The $A m m 2$ phase } \\
\hline 1 & 91 & 66 & 87 & 89 & $60 i$ & $54 i$ \\
\hline 2 & 76 & 46 & 84 & 79 & $69 i, 39 i$ & $72 i, 41 i$ \\
\hline \multirow[t]{3}{*}{3} & 61 & 36 & 68 & 77 & $79 i, 58 i, 46 i, 38 i, 25 i$ & $79 i, 53 i, 20 i, 16 i$ \\
\hline & $\Gamma(0,0,0)$ & $Z\left(0,0, \frac{1}{2}\right)$ & $X / Y$ & $U / T$ & $S$ & $R$ \\
\hline & \multicolumn{6}{|c|}{ The $P m c 2_{1}$ phase } \\
\hline 1 & 84 & 66 & $70 / 76$ & $78 / 82$ & 86 & 89 \\
\hline 3 & 60 & 35 & $67 / 73$ & $68 / 74$ & 80 & 81 \\
\hline
\end{tabular}

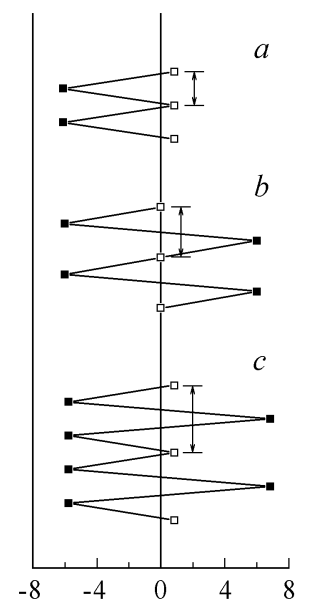

Rotation angle (deg.)

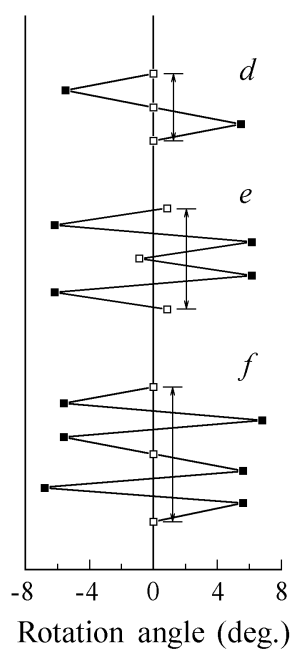

FIG. 3. The octahedra rotation angles in the ground state of $\left(\mathrm{BaTiO}_{3}\right)_{1}\left(\mathrm{BaZrO}_{3}\right)_{n}$ superlattices corresponding to the condensation of phonons at the $(a-c) M$ and $(d-f) A$ points. $(a, d) n=1(L=2),(b, e) n=2(L=3),(c, f) n=3(L=4)$. The $\mathrm{BaTiO}_{3}$ and $\mathrm{BaZrO}_{3}$ layers are denoted by open and filled squares, respectively. Vertical lines with arrows indicate the physical periods of the resulting structures.

$L$ specified by the layer sequence. Modes with lower instability (Table I) are qualitatively similar to the abovedescribed modes at the $M$ point.

An abrupt change in the rotation angle in going from the $\mathrm{BaTiO}_{3}$ layer to the $\mathrm{BaZrO}_{3}$ layer and its weak dependence on the period of SL correlate with the small change of the unstable phonon frequency along the $M-A$ line, which indicates a strong localization of these rotations in the layers.

To search for the ground state of SLs, the oxygen octa-
TABLE II. The lattice parameters in the paraelectric $P 4 / m m m$ phase of free-standing $\left(\mathrm{BaTiO}_{3}\right)_{1}\left(\mathrm{BaZrO}_{3}\right)_{n}$ superlattices with $1 \leq n \leq 7$, the energy of the ferroelectric ground state $\Delta E_{1}$ (the $A m m 2$ phase), the energy $\Delta E_{2}$ of the $I 4 / \mathrm{mcm}$ or $\mathrm{P} 4 / \mathrm{mbm}$ phases with pure rotations, the energy of the true ground state $\Delta E_{3}$ (Ima2 or $P m c 2_{1}$ phase), and the polarization in the ferroelectric ground $\left(P_{s 1}\right)$ and true ground $\left(P_{s 3}\right)$ states.

\begin{tabular}{ccccccc}
\hline \hline$n$ & 1 & 2 & 3 & 4 & 5 & 7 \\
\hline$a_{0}(\mathrm{Bohr})$ & 7.7182 & 7.7747 & 7.8009 & 7.8160 & 7.8258 & 7.8379 \\
$c_{0}(\mathrm{Bohr})$ & 15.3298 & 23.1923 & 31.0604 & 38.9304 & 46.8013 & 62.5443 \\
$\Delta E_{1}(\mathrm{meV})$ & -72.7 & -101.0 & -115.1 & -123.6 & -129.3 & -136.2 \\
$\Delta E_{2}(\mathrm{meV})$ & -21.3 & -34.6 & -46.7 & -57.7 & -68.3 & -89.2 \\
$\Delta E_{3}(\mathrm{meV})$ & -78.3 & -116.8 & -142.3 & -161.5 & -177.8 & -205.3 \\
$P_{s 1}\left(\mathrm{C} / \mathrm{m}^{2}\right)$ & 0.2755 & 0.2024 & 0.1568 & 0.1273 & 0.1070 & - \\
$P_{s 3}\left(\mathrm{C} / \mathrm{m}^{2}\right)$ & 0.2591 & 0.1859 & 0.1445 & 0.1113 & 0.0984 & - \\
\hline \hline
\end{tabular}

hedra rotations corresponding to the least stable phonons at the $M$ and $A$ points of the Brillouin zone were added to the polar Amm2 structure. A comparison of the total energies of the structures resulting from simultaneous condensation of the "rotational" and ferroelectric modes shows that the ground-state structure depends on the superlattice period $L$ : for odd $L$, it is described by the space group Ima2 and results from the condensation of the unstable "rotational" mode at the $A$ point; for even $L$, it is described by the space group $P m c 2_{1}$ and results from the condensation of the unstable "rotational" mode at the $M$ point. The energy difference of the structures resulting from the condensation of phonons at the $M$ and $A$ points is rather small $(1.6-1.9 \mathrm{meV})$. The energies of the structures corresponding to the ferroelectric ground state and to the true ground state, as well as to nonpolar structures with pure octahedra rotations (space group 


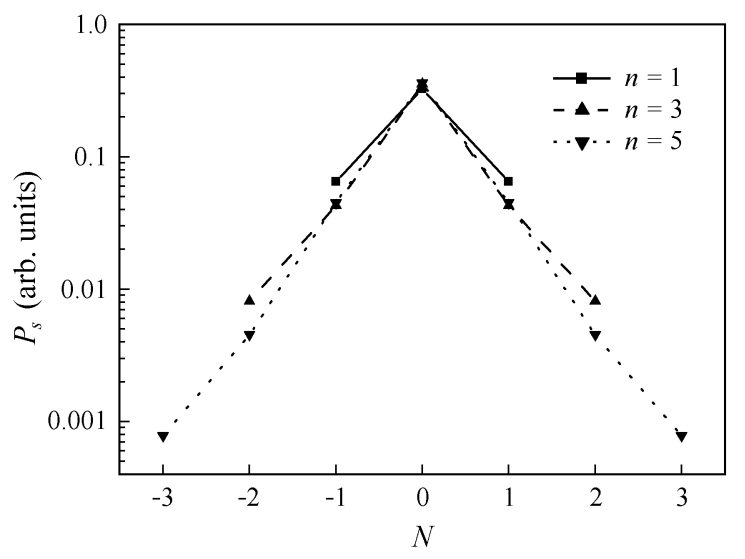

FIG. 4. The polarization distribution between the layers in the ground-state structure of $\left(\mathrm{BaTiO}_{3}\right)_{1}\left(\mathrm{BaZrO}_{3}\right)_{n}$ superlattices with $n=1,3$, and 5 . The layer number $N=0$ corresponds to the $\mathrm{BaTiO}_{3}$ layer.

$I 4 / \mathrm{mcm}$ for odd $L$ and $P 4 / \mathrm{mbm}$ for even $L$ ) are given in Table $\prod^{22}$ An increase in all three energies with increasing $n$ is due to two effects: (i) an increase in the $\mathrm{BaZrO}_{3}$ volume fraction in the structure, which results in an increase of the instability of SLs with respect to the octahedra rotation; (ii) an increase in biaxial tension of the $\mathrm{BaTiO}_{3}$ layers (as a consequence of an increase in the in-plane lattice parameter), which enhances the ferroelectric instability. The corresponding change in the frequencies of unstable phonons at the $\Gamma$ point can be seen in Table 【

The polarization profiles in the obtained ground states for SLs with $n=1,3$, and 5 are shown in Fig. 4. One can see that the polarization is concentrated in the barium titanate layer and almost exponentially decreases with distance with a characteristic length scale of $\sim 2.0 \AA$. It is interesting that the spontaneous polarization in the true ground state is lower than the polarization in the ferroelectric ground state by only $6-12 \%$ (Table II). This indicates the weak effect of the octahedra rotations on the spontaneous polarization in SLs ${ }^{23}$ We explain this by the fact that regions with high polarization in the SLs under consideration are spatially separated from regions with strong octahedra rotations, and thus the competition between the structural and ferroelectric instabilities becomes significantly decreased. This feature essentially distinguishes the superlattices from such crystals as $\mathrm{CaTiO}_{3}$ and $\mathrm{PbTiO}_{3}$ with competing instabilities in the cubic phase. In the former case, the structural distortions completely suppress ferroelectricity; in the latter case, an opposite effect occurs.

To test the possibility of forming the arrays of quasitwo-dimensional polarized planes in the ground state of $\left(\mathrm{BaTiO}_{3}\right)_{1}\left(\mathrm{BaZrO}_{3}\right)_{n}$ SLs, we estimated the interlayer interaction energy. The energy $2 W_{\text {int }}$ (the energy corresponding to two domain walls) was calculated as the difference of the total energies of ferroelectrically and an- tiferroelectrically ordered SLs with a doubled period in a similar way as in Ref. 4; in this calculation, the octahedra rotations were neglected. The value of $2 W_{\text {int }}$ was $7.46 \mathrm{meV}$ for $n=1,1.505 \mathrm{meV}$ for $n=2$, and $0.420 \mathrm{meV}$ for $n=3$, and decreased almost exponentially with increasing $n$. For all $n$, this energy was much lower than the energy gain resulting from the ferroelectric ordering $\Delta E_{1}$ (Table III), which ensures almost independent polarizations in the quasi-two-dimensional layers and the formation of the arrays of independent polarized planes similar to those observed in Ref. 4. The potential barrier height $\Delta U$ for reorientation of the polarization, which occurs in the SLs under consideration by rotating the polarization vector in the layer plane, was $22.9 \mathrm{meV}$ (per Ti atom) for $n=1,33.2 \mathrm{meV}$ for $n=2$, and $38.3 \mathrm{meV}$ for $n=3$. The obtained potential barrier heights and ferroelectric ordering energies are much higher than those for the $\mathrm{KNbO}_{3} / \mathrm{KTaO}_{3}$ SLs considered in Refs. 4 and $\underline{5}$. In our opinion, these values are sufficiently large to provide stable polarization in the arrays of quasi-two-dimensional polarized planes at $300 \mathrm{~K}$.

\section{DISCUSSION}

We start the discussion with an analysis of possible polar structures in $\left(\mathrm{BaTiO}_{3}\right)_{1}\left(\mathrm{BaZrO}_{3}\right)_{n}$ SLs. The ferroelectric instability in the ...-Ti-O $-\ldots$ chains can lead not only to the ferroelectric ordering, but also to the formation of antiferroelectric structures resulting from the condensation of unstable phonons at $Z, R$, and $X$ points at the boundary of the Brillouin zone. Three types of the polarization ordering in the chains, which result from the condensation of these unstable phonons in the paraelectric $P 4 / \mathrm{mmm}$ phase, are shown in Fig. 5. It is seen that these structures differ only in the relative orientation of polarization in neighboring chains. For example, for the phonon condensation at the $X$ point, the antiferroelectric Pmma phase appears in the SLs; the modulation wave vector in this phase is directed along the $x$ axis, whereas the out-of-phase displacements of $\mathrm{Ti}$ and $\mathrm{O}$ atoms forming the chains are directed along the $y$ axis (Fig. 5, b). It is remarkable that this phase still exhibits the ferroelectric instability with respect to the polar displacements along the $x$ axis and finally transforms to the polar $P m$ $\left(P m c 2_{1}\right.$ for $\left.n=1\right)$ phase being ferrielectric. Similarly, the antiferroelectric $C m m a$ phase with the modulation described by the phonon at the $R$ point and with outof-phase displacements along the $y$ axis $($ Fig. 5 c) also is ferroelectrically unstable and finally transforms to the ferrielectric Abm2 phase. In contrast to non-degenerate unstable phonons at the $X$ and $R$ points, the unstable phonon at the $Z$ point is doubly degenerate, and its condensation can lead to two antiferroelectric phases, Pmma (Fig. 15, $d$ ) and $\mathrm{Cmcm}$, with displacements in one or both ...-Ti-O-... chains, respectively. These displacements result in the in-plane microscopic polarization directed along [100] and [110]. It was the latter of these two phases 

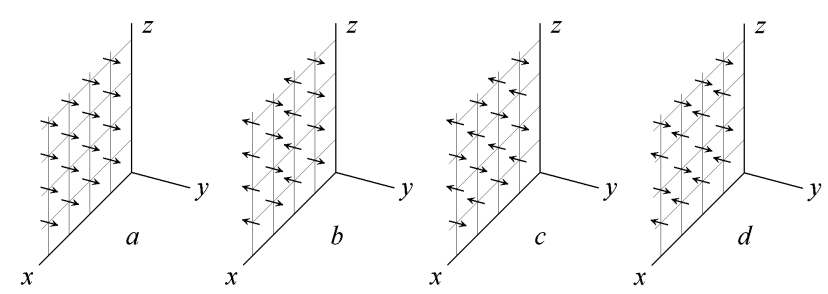

FIG. 5. Patterns of the polarization ordering in the ...-Ti-O... chains propagating along the $y$ axis which accompany the condensation of unstable phonon at the $(a) \Gamma,(b) X,(c) R$, and $(d) Z$ points.

TABLE III. Energies $E$ (in $\mathrm{meV}$ ) of the antiferroelectric phases arising from the condensation of unstable phonons at the boundary of the Brillouin zone and of the ferroelectric $A m m 2$ phase for free-standing $\left(\mathrm{BaTiO}_{3}\right)_{1}\left(\mathrm{BaZrO}_{3}\right)_{n}$ superlattices with $n=1,2$, and 3 . The energy of the paraelectric $\mathrm{P} 4 / \mathrm{mmm}$ is taken as the energy reference.

\begin{tabular}{ccccccc}
\hline \hline \multirow{2}{*}{$\begin{array}{c}\text { Unstable } \\
\text { phonon }\end{array}$} & \multicolumn{2}{c}{$n=1$} & \multicolumn{2}{c}{$n=2$} & \multicolumn{2}{c}{$n=3$} \\
\cline { 2 - 7 } & Phase & $E$ & Phase & $E$ & Phase & $E$ \\
\hline$X$ & $P m m a$ & -37.3 & $P m m a$ & -52.1 & $P m m a$ & -59.8 \\
& $P m c 2_{1}$ & -68.1 & $P m$ & -95.2 & $P m$ & -109.0 \\
$R$ & $C m m a$ & -33.7 & $C m m a$ & -51.1 & $C m m a$ & -59.7 \\
& $A b m 2$ & -64.9 & $A b m 2$ & -94.3 & $A b m 2$ & -108.8 \\
$Z$ & $P m m a$ & -41.9 & $P m m a$ & -60.7 & $P m m a$ & -69.6 \\
& $C m c m$ & -68.9 & $C m c m$ & -100.3 & $C m c m$ & -114.9 \\
$\Gamma$ & $A m m 2$ & -72.7 & $A m m 2$ & -101.0 & $A m m 2$ & -115.1 \\
\hline \hline
\end{tabular}

which was considered above in the calculation of the interlayer interaction energy.

The ferroelectric instability of the intermediate Pmma and $C m m a$ phases is easily understandable: in these two phases, the structural relaxation occurs in only one of two ferroelectrically unstable $\ldots-\mathrm{Ti}-\mathrm{O}-\ldots$ chains propagating in the [100] and [010] directions, and so the structure retains the ferroelectric instability in the second chain. In the $P m c 2_{1}, P m, A b m 2$, and $C m c m$ phases, the structural relaxation occurs simultaneously in both chains; this is why these structures are stable (metastable). In all cases, the obtained intermediate and ferrielectric phases have higher energies in comparison with the ferroelectric Amm2 phase (Table 【II).

Now we discuss the structure of rotations in the SLs under consideration. A comparison of the frequencies of unstable phonons corresponding to various combinations of rotations in the layers of SLs with the same period shows that phonons with out-of-phase rotations in neighboring layers are energetically least stable. These rotations are fully consistent with the instability at the $R$ point in the parent cubic $\mathrm{BaZrO}_{3}$ (Fig. 11). The main difference between the two rotation systems corresponding to unstable phonons at the $M$ and $A$ points is the relation between rotations in the neighboring periods of the superlattice: for the phonon condensation at the $M$ point, when the structure is translated by one SL period, the octahedra are rotated in phase; in the case of the phonon condensation at the $A$ point, they are rotated out of phase. It can be seen that the ground state of the SL always corresponds to such a rotation system in which the octahedra are rotated out of phase in any pair of neighboring layers, i.e., the $M$ phonon is condensed in the SLs with even period and the $A$ phonon is condensed in the SLs with odd period.

One more subject for discussion is the comparison of the obtained results with the experimental data of Ref $\frac{15}{}$. According to the X-ray data from this work, the spontaneous polarization in SLs is predominantly oriented in the layer plane; however, the line narrowing and an increase in the frequency of the soft $E$ mode in Raman spectra indicate the monoclinic distortion of the structure and the appearance of the $z$-component of polarization in $\mathrm{BaTiO}_{3}$ layers. The $z$-component of polarization determined from the hysteresis loops nonmonotonically depends on the superlattice period. The authors supposed that the appearance of the polarization component normal to the film is caused by the ferroelectric phase transition predicted in Ref. 6 in biaxially compressed $\mathrm{BaZrO}_{3}$ layers.

The calculations performed in the present work showed that the $A_{2 u}$ phonon is indeed unstable in biaxially compressed $\mathrm{BaZrO}_{3}$ (space group $P 4 / \mathrm{mmm}$ ) with the in-plane lattice parameter equal to the lattice parameter of the free-standing $\left(\mathrm{BaTiO}_{3}\right)_{1}\left(\mathrm{BaZrO}_{3}\right)_{1} \mathrm{SL}\left(a_{0}=\right.$ 7.7182 Bohr) (according to our data, the critical lattice parameter at which the instability of this phonon appears is $\approx 7.735$ Bohr and notably exceeds the value of 7.425 Bohr reported in Ref. 6). However, the instability of the $A_{2 u}$ phonon in biaxially stretched $\mathrm{BaTiO}_{3}$ with the lattice parameter equal to $a_{0}$ is absent (the phonon frequency is $45 \mathrm{~cm}^{-1}$ ). Therefore, the appearing of the $z$-component of polarization in SLs needs a more detailed analysis.

As we have shown previously,$\underline{3}$ the $A_{2 u}$ mode (which is responsible for the appearance of the $z$-component of polarization) is stable $\left(37 \mathrm{~cm}^{-1}\right)$ in the paraelectric $P 4 / \mathrm{mmm}$ phase of the freestanding $\left(\mathrm{BaTiO}_{3}\right)_{1}\left(\mathrm{BaZrO}_{3}\right)_{1}$ SL. The calculations of the phonon frequencies for the $P 4 / \mathrm{mmm}$ phase of $\left(\mathrm{BaTiO}_{3}\right)_{n}\left(\mathrm{BaZrO}_{3}\right)_{n}$ SLs with $n=2$, 3, and 4 performed in this work showed that the frequency of this mode rapidly decreases with increasing $n\left(28.5 \mathrm{~cm}^{-1}\right.$ for $n=2$ and $17 \mathrm{~cm}^{-1}$ for $n=3$ ) and becomes unstable at $n=4\left(7 i \mathrm{~cm}^{-1}\right)$. However, the instability of the $A_{2 u}$ phonon in the paraelectric phase does not mean that the $z$-component of polarization will appear in the ferroelectric ground state. The in-plane spontaneous polarization, which appears due to the strong instability of the $E_{u}$ phonon, abruptly increases the frequency of the corresponding mode $\left(B_{1}\right.$ or $\left.B_{2}\right)$ in the $A m m 2$ phase, and this phase becomes stable with respect to the appearance of the $z$-component of polarization.

This stability reduction of the $A_{2 u}$ phonon with increasing $n$ recalls the results obtained for the 
$\left(\mathrm{BaTiO}_{3}\right)_{m}(\mathrm{BaO})_{n}$ Ruddlesden-Popper SLs $\stackrel{24}{=}$ In these SLs, a similar decrease in the $A_{2 u}$ phonon frequency with increasing the $\mathrm{BaTiO}_{3}$ layer thickness was observed; at $m \geq 8$, it became unstable. However, already at $m=4$, the instability at the $X$ point appearing in the SLs resulted in the formation of domain-like structure, since it was energetically more favorable to form a structure with a $z$-component of polarization periodically varying in space, rather than to uniformly polarize the $\mathrm{BaO}$ layer having a low dielectric constant. The macroscopic $z$ component of polarization is absent in such a structure.

Another possible cause for the appearance of the $z$ component of polarization can be a substrate-induced strain in the superlattice (in Ref. 15, the SLs were grown on $\mathrm{MgO}$ substrate). Calculations show that in $\left(\mathrm{BaTiO}_{3}\right)_{n}\left(\mathrm{BaZrO}_{3}\right)_{n}$ SLs with $n=1$ and 2, for the inplane lattice parameter equal to $a=0.99 a_{0}$ and $0.98 a_{0}$ ( $a_{0}$ is the in-plane lattice parameter of the free-standing SL) and the disabled octahedra rotations, the polarization vector is rotated by $\sim 38^{\circ}$ and $\sim 67^{\circ}$ from the layer plane (the space group of the ferroelectric ground state is $C m$ ); for $a=0.97 a_{0}$ and disabled rotations, the vector is rotated by $90^{\circ}$ (the space group of the ferroelectric ground state is $P 4 \mathrm{~mm}$ ). However, when the octahedra rotations are enabled, in all SLs we see an unexpected result: the $z$-component of polarization disappears in SLs with $a=0.99 a_{0}$ and $0.98 a_{0}$ (space group of the ground state is $P m c 2_{1}$ for $n=1$ and $P n c 2$ for $n=2$ ), whereas in the SL with $a=0.97 a_{0}$, the polarization is rotated toward the layer plane by $\sim 24^{\circ}$ (the space group of the ground state is $P c$ ).

The competition between the ferroelectric and structural instabilities in crystals with the perovskite structure has been known for a long time $; \stackrel{25,26}{~ h o w e v e r, ~ t h e ~}$ fact that the octahedra rotations can have such a strong effect on the polarization seems to be observed for the first time. This finding can be important for the following reason. In the SLs under consideration, the energy gain resulting from the structural distortions is less than the energy gain resulting from the ferroelectric ordering (compare the energies $\Delta E_{1}$ and $\Delta E_{2}$ in Table II). This leads us to expect that, as the temperature increases, the structural phase transition in the SLs will occur at a lower temperature than the ferroelectric phase transition. Then, since the disappearance of the octahedra rotations results in the onset of the $z$-component of polarization, the situation is possible that an improper ferroelectric phase transition accompanied by the appearance of a nonzero $z$-component of polarization can occur in the SLs when the structural phase transition temperature is approached from below (when the octahedra rotation angle rapidly decreases). The abrupt rotation of the polarization vector as the temperature varies can result in the appearance of a number of physical property anomalies resembling the anomaly in the piezoelectric coefficients near the morphotropic phase boundary. However, currently, it is not clear whether the structural phase transition occurs in $\mathrm{BaZrO}_{3}$. Nevertheless, even if the establishment of the long-range order in the octahedra rotations in $\mathrm{BaZrO}_{3}$ is impossible for some reason, the changes in the local angles of the rotations with temperature can have a significant effect on the $z$-component of polarization.

The above-considered attempts to explain the appearance of the $z$-component of polarization, which were based on the assumption of a uniformly strained $\mathrm{BaTiO}_{3} / \mathrm{BaZrO}_{3} \mathrm{SL}$, were not successful (the strong biaxial substrate-induced in-plane compression of the SL cannot be retained in a thick SL). It seems that the only way to explain this phenomenon is to consider the stress relaxations occurring in SLs with thick layers. This idea has already been used to explain the appearance of the $z$ component of polarization in $\mathrm{BaTiO}_{3} / \mathrm{BaZrO}_{3} \mathrm{SLs}$ with large periods,$\frac{3,13}{1}$ Our calculations show that the critical in-plane lattice parameter at which the $z$-component of polarization appears in $\mathrm{BaTiO}_{3}$ is $\sim 7.565 \mathrm{Bohr}$; in this case, the out-of-plane lattice parameter is $c \approx 7.471 \mathrm{Bohr}$. If we take into account the systematic error $(-0.7 \%)$ in the prediction of the lattice parameters for $\mathrm{BaTiO}_{3}$, the experimental parameter $c=3.981 \AA$ in the $\mathrm{BaTiO}_{3}$ layer should correspond to this situation. According to the Xray data, $\stackrel{15}{=}$ such a relaxation of mechanical stress already appears in the SL with a period of $L=32 \AA$. So, this relaxation explains the appearance of the $z$-component of polarization in SLs with the specified and larger periods.

The first-principles calculations allow us to understand why the quality of short-period $\mathrm{BaTiO}_{3} / \mathrm{BaZrO}_{3}$ and $\mathrm{BaTiO}_{3} / \mathrm{Ba}(\mathrm{Ti}, \mathrm{Zr}) \mathrm{O}_{3}$ SLs grown in the [001] direction is not very good 15,27 This is because of the tendency of $\mathrm{BaTi}_{1-x} \mathrm{Zr}_{x} \mathrm{TiO}_{3}$ solid solutions to three-dimensional chessboard-type ordering of cations at the $B$ sites. The calculations show that the energy of the paraelectric phase of the $\left(\mathrm{BaTiO}_{3}\right)_{1}\left(\mathrm{BaZrO}_{3}\right)_{1}$ SL with the elpasolite structure (space group $F m 3 m$ ), which appears when growing the SL in the [111] direction, is by $83 \mathrm{meV}$ (per formula unit) lower than the energy of the same SL but grown in the [001] direction. It is possible that defects generated during the stress relaxation in SLs grown in the [001] direction become the nucleation centers of threedimensional chessboard-type ordered inclusions. Furthermore, the possible electrical activity of these defects can be a factor which changes the electrostatic boundary conditions at the heterointerface and stabilizes the polar state in the $\mathrm{BaTiO}_{3}$ layer without inducing an appreciable electric field and polarization in the $\mathrm{BaZrO}_{3}$ layer.

\section{CONCLUSION}

The properties of $\mathrm{BaTiO}_{3} / \mathrm{BaZrO}_{3}$ superlattices with competing ferroelectric and structural instabilities were calculated from first principles within the density functional theory. It was established that the quasi-twodimensional ferroelectricity with the polarization oriented in the layer plane, which weakly interacts with the polarization in neighboring layers, occurs in SLs grown 
in the [001] direction with one-unit-cell thick barium titanate layer. It was shown that the energy gain from the ferroelectric ordering and the height of the potential barrier separating different orientational states of polarization are sufficiently large to observe the formation of an array of independent polarized planes at room temperature. The effect of the structural instability on the properties of SLs was considered. It was shown that the ground state is a result of simultaneous condensation of the ferroelectric $\Gamma_{15}$ mode and phonons at the $M$ point (for SLs with even period) or at the $A$ point (for SLs with odd period). Thus, the ground state is a structure with out-of-phase rotations in neighboring layers, in which highly polarized layers and layers with strong octahedra rotations are spatially separated. This significantly reduces the influence of structural distor- tions on the spontaneous polarization. It was shown that the switching on of the octahedra rotations in biaxially compressed $\mathrm{BaTiO}_{3} / \mathrm{BaZrO}_{3}$ SLs with an in-plane strain of $1-3 \%$ results in an abrupt change in the polarization direction. This suggests that an improper ferroelectric phase transition accompanied by the appearance of a nonzero $z$-component of polarization can occur in these SLs with increasing temperature.

\section{ACKNOWLEDGMENTS}

The calculations presented in this work were performed on the laboratory computer cluster (16 cores) and the SKIF-MGU ("Chebyshev") and "Lomonosov" supercomputers.
* swan@scon155.phys.msu.ru

1 K. M. Rabe, Curr. Opin. Solid State Mater. Sci. 9, 122 (2005).

2 P. Ghosez and J. Junquera, in Handbook of Theoretical and Computational Nanotechnology, vol. 9, edited by M. Rieth and W. Schommers (American Scientific Publishers, 2006) pp. $623-728$.

3 A. I. Lebedev, Phys. Solid State 52, 1448 (2010)

4 A. I. Lebedev, Phys. Solid State 53, 2463 (2011)

5 A. I. Lebedev, Phys. Status Solidi B 249, 789 (2012)

${ }^{6}$ O. Diéguez, K. M. Rabe, and D. Vanderbilt, Phys. Rev. B 72, 144101 (2005).

7 A. R. Akbarzadeh, I. Kornev, C. Malibert, L. Bellaiche, and J. M. Kiat, Phys. Rev. B 72, 205104 (2005)

8 J. W. Bennett, I. Grinberg, and A. M. Rappe, Phys. Rev. B 73, 180102(R) (2006).

9 A. Bilić and J. D. Gale, Phys. Rev. B 79, 174107 (2009)

10 T. Tsurumi, T. Ichikawa, T. Harigai, H. Kakemoto, and S. Wada, J. Appl. Phys. 91, 2284 (2002).

11 T. Harigai, S.-M. Nam, H. Kakemoto, S. Wada, K. Saito, and T. Tsurumi, Thin Solid Films 509, 13 (2006)

12 T. Harigai and T. Tsurumi, Ferroelectrics 346, 56 (2007)

13 P. R. Choudhury and S. B. Krupanidhi, Appl. Phys. Lett. 92, 102903 (2008)

14 P. R. Choudhury and S. B. Krupanidhi, J. Appl. Phys. 104, 114105 (2008)

15 M. E. Marssi, Y. Gagou, J. Belhadi, F. D. Guerville, Y. I. Yuzyuk, and I. P. Raevski, J. Appl. Phys. 108, 084104 (2010)

16 X. Gonze, B. Amadon, P.-M. Anglade, J.-M. Beuken, F. Bottin, P. Boulanger, F. Bruneval, D. Caliste, R. Caracas, M. Côté, T. Deutsch, L. Genovese, P. Ghosez, M. Giantomassi, S. Goedecker, D. R. Hamann, P. Hermet,
F. Jollet, G. Jomard, S. Leroux, M. Mancini, S. Mazevet, M. J. T. Oliveira, G. Onida, Y. Pouillon, T. Rangel, G.-M. Rignanese, D. Sangalli, R. Shaltaf, M. Torrent, M. J. Verstraete, G. Zerah, and J. W. Zwanziger, Computer Phys. Commun. 180, 2582 (2009)

17 "Opium-pseudopotential generation project," http://opium.source

18 A. I. Lebedev, Phys. Solid State 51, 362 (2009).

19 EXAFS measurements of the Debye-Waller factor for $\mathrm{Ba}-$ $\mathrm{O}$ bonds in $\mathrm{BaZrO}_{3}$ revealed its anomalously high values at $300 \mathrm{~K}$, corresponding to the amplitude of the local rotations of $\sim 4$ degrees. In our opinion, this gives an evidence of the instability under consideration. The results of these studies will be published elsewhere.

${ }^{20}$ R. Yu and H. Krakauer, Phys. Rev. Lett. 74, 4067 (1995)

21 Hereafter, we shall use the notation of the points in the Brillouin zone of the tetragonal paraelectric structure for their notation in the low-symmetry structures.

22 The ferroelectric ground state is a structure with a minimum energy obtained by taking into account only the ferroelectric unstable mode.

23 An additional cause of the decrease in the polarization in free-standing SLs when the octahedra rotations are switched on can be a systematic decrease (by 0.02 0.03 Bohr) in the in-plane lattice parameter.

24 E. Bousquet, J. Junquera, and P. Ghosez, Phys. Rev. B 82, 045426 (2010)

25 W. Zhong and Phys. Rev. Lett. 74, 2587 (1995)

26 D. Vanderbilt and Ferroelectrics 206-207, 181 (1998).

D. Vanderbilt, W. Zhong,

27 F. De Guerville, M. El Marssi, I. P. Raevski, M. G. Karkut, and Y. I. Yuzyuk, Phys. Rev. B 74, 064107 (2006). 